\title{
NEW DATA OF TWO Mylonchulus SPECIES (Mononchida: Mylonchulidae) AND AN UPDATED KEY TO SPECIES FROM VIETNAM
}

\author{
Vu Thi Thanh Tam \\ Institute of Ecology and Biological Resources, VAST
}

\begin{abstract}
Two species of Mylonchulus, M. kermaniensis and M. oceanicus, were recorded, described and illustrated for the first time from Vietnam. Mylonchulus kermaniensis was collected from Nghe An province, their measurements and description correspond well with the type population from Kerma, Iran except for the absence of vulval papilla(e). Mylonchulus oceanicus was found in Cao Loc, Lang Son province. Measurements and description of the Vietnamese specimens fit well the type population from Hawaii, USA as well as another population from Japan except for a slightly shorter tail ( $c=47-56$ vs 39-49 in type and other population). In addition, an updated key to twenty already known species in Vietnam is also given in this paper.
\end{abstract}

Keywords: Mylonchulidae, Mylonchulus, new records, nematode, Vietnam.

Citation: Vu Thi Thanh Tam, 2016. New data of two Mylonchulus species (Mononchida: Mylonchulidae) and an updated key to species from Vietnam. Tap chi Sinh hoc, 38(3): 287-292. DOI: 10.15625/0866$7160 / \mathrm{v} 38 \mathrm{n} 3.7924$.

*Corresponding author: vtam7572@yahoo.com

\section{INTRODUCTION}

In Vietnam, up to now, the studies on predaceous nematodes of the order Mononchida have revealed 18 species on a total of 77 species of the genus Mylonchulus (Mylonchulidae) [4]. In the present paper, two additional Mylonchulus species were identified from Nghe An and Cao Bang provinces; they represent new records for the nematode fauna in Vietnam. M. kermaniensis was collected from soil around the base of banana at Nghe An province. This species was originally described from Kerman and Iran [6].

Andrassy (1986) [3] first reported M. oceanicus from Hawaii, USA and Ahmad et al. (2010) [2] recorded this species from Okinawa, Japan though with a slightly shorter tail than in the type population. In present paper, M. oceanicus is recorded from Cao Loc (Lang Son province).

\section{MATERIALS AND METHODS}

Soil samples were collected from natural forest areas in Nghe An and Lang Son provinces (Vietnam). Nematodes were extracted from soil samples by modified Baermann funnel technique [7], killed by heat, fixed in formaldehyde $4 \%$, transferred to anhydrous glycerol according to Seinhorst (1959) [5], and mounted on glass slides for microscopic observation. Permanent slides were stored at the Department of Nematology, IEBR. Figures were drawn using an Olympus microscope $\mathrm{CH} 40$ with drawing tube and illustrations were edited by Adobe Illustrator CS6.

IEBR stands for Institute of Ecology and Biological Resources; VAST stands for Vietnam Academy of Science and Technology.

\section{RESULTS AND DISCUSSION}

\section{Mylonchulus kermaniensis Shokoohi, Mehrabi-Nasab, Mirzaei \& Peneva, 2013 (Figs. 1A-C)}

Material: 6 females in good condition. Measurements: see table 1.

Female: Moderately slender nematodes of medium size, 1.2-1.5 mm long. Habitus after fixation ventrally arcuate, particularly toward posterior end. Under light microscope: cuticle smooth, 2-3 $\mu \mathrm{m}$ thick at the base of oesophagus. Lip region offset from the body contour by slight depression, its width 22-25 $\mu \mathrm{m}$. Buccal cavity medium size, 24-26.5 $\mu \mathrm{m}$ long by $14-15.8 \mu \mathrm{m}$ wide, funnel shaped, tapering at base; its wall 
strongly sclerotized,. Amphideal fovea cupshaped. Dorsal tooth massive, with sharp apex, pointing forward. Apex of dorsal tooth situated at $77-84 \%$ of buccal cavity length from its base. Small rasp-like denticles arranged in six transverse rows, without teeth in ventral wall.
Oesophagus cylindroid, 336-391 $\mu \mathrm{m}$ long, nerve ring at $40-42 \%$ of its length from anterior end; secretory-excretory pore and system not seen. Oesophago-intestinal junction nontuberculate. Cardia projecting into intestinal lumen.

Table 1. Morphometric data of Mylonchulus kermaniensis Shokoohi, Mehrabi-Nasab, Mirzaei \& Peneva, 2013

\begin{tabular}{lcc}
\hline \multirow{2}{*}{ Local } & \multicolumn{2}{c}{ Mylonchulus kermaniensis } \\
\cline { 2 - 3 } & Types Kerman, Iran & Nghe An, Vietnam \\
\hline $\mathrm{n}$ & Shokoohi et al. (2013) & Present paper \\
\hline $\mathrm{L}(\mathrm{mm})$ & 6 q & 6 क \\
$\mathrm{a}$ & $1.28-1.49$ & $1.2-1.5$ \\
$\mathrm{~b}$ & $23.7-34.5$ & $29-35.5$ \\
$\mathrm{c}$ & $3.4-3.7$ & $3.5-3.9$ \\
$\mathrm{c}$ & $27.9-38.9$ & $35-41$ \\
$\mathrm{~V}(\%)$ & $1.3-1.7$ & $1.3-1.6$ \\
Buccal cavity length $(\mu \mathrm{m})$ & $61-66$ & $60-64$ \\
Buccal cavity width $(\mu \mathrm{m})$ & $24-27$ & $24-26.5$ \\
Apex of dorsal tooth position & $15-18$ & $14-15.8$ \\
from base of buccal cavity $(\%)$ & $66-82$ & $77-84$ \\
Lip region width $(\mu \mathrm{m})$ & $21.5-25$ & $22-24.6$ \\
Neck length $(\mu \mathrm{m})$ & $357-407$ & $336-391$ \\
Body width $(\mu \mathrm{m})$ & $41-57$ & $40.5-45$ \\
Anal body width $(\mu \mathrm{m})$ & $28-32$ & $25.5-31$ \\
Tail length $(\mu \mathrm{m})$ & $37-49$ & $35-39.5$ \\
Rectum $(\mu \mathrm{m})$ & $19-25$ & $16.7-24.6$ \\
\hline
\end{tabular}

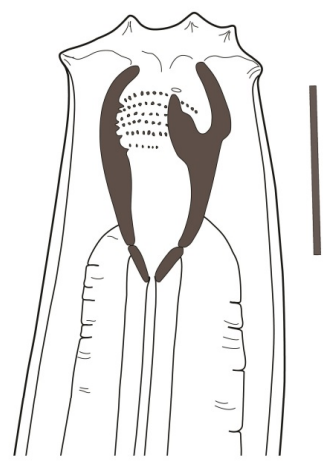

A

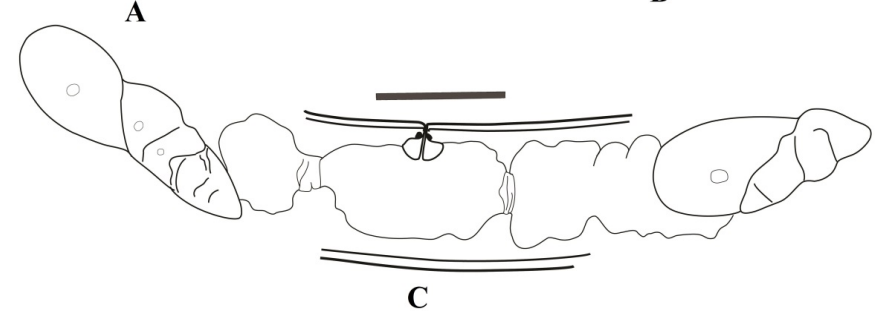

Figure 1. Mylonchulus kermaniensis Shokoohi, MehrabiNasab, Mirzaei \& Peneva, 2013

A. Head region; B. Female tail region; C. Female reproductive system (Scale bars: A, B $=20$ $\mu \mathrm{m} ; \mathrm{C}=40 \mu \mathrm{m})$ 
Reproductive system didelphicamphidelphic, both branches equally developed with ovary reflexed. Oocytes arranged in single row. Sphincter present between oviduct and uterus. Vulva, a transverse slit in ventral view, not protruding. Vagina short with pars refringens vaginae sclerotized, visible as 2 pieces in optical section. Advulval papilla(e) absent. Rectum straight, thick-walled and muscular, 17-25 $\mu \mathrm{m}$, prerectum not seen. Tail sigmoid, sharply bend ventrad with digital posterior portion clearly bend dorsal side; 35-40 $\mu \mathrm{m}$ long or 1.3-1.6 anal body diameter. Caudal gland moderately developed and spinneret opening prominent, terminal.

Male: Not found.

Remark: The measurements and description of Vietnamese specimens correspond well with holotype and paratype specimens from type population from Kerma, Iran [6] with the exception of the absence of advulval papilla(e) in all specimens.

\section{Locality: Nghe An.}

\section{Mylonchulus oceanicus Adrassy, 1986 (Figs. 2A-C)}

Material: 10 females in good condition. Measurements: see table 2.
A

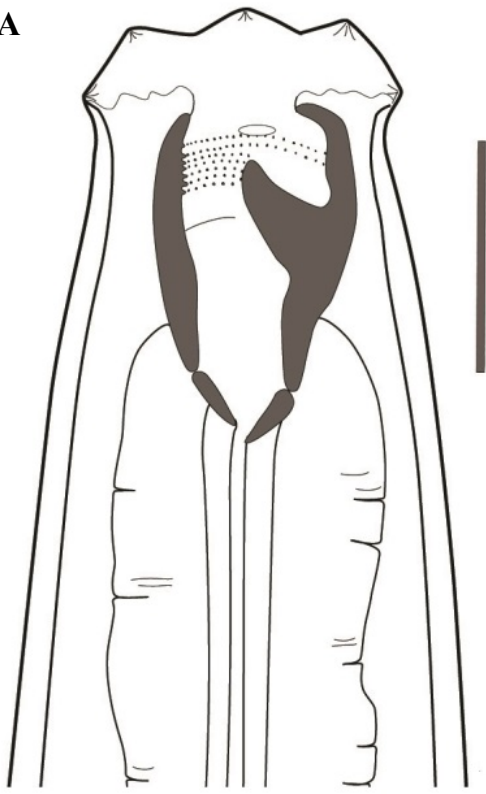

B

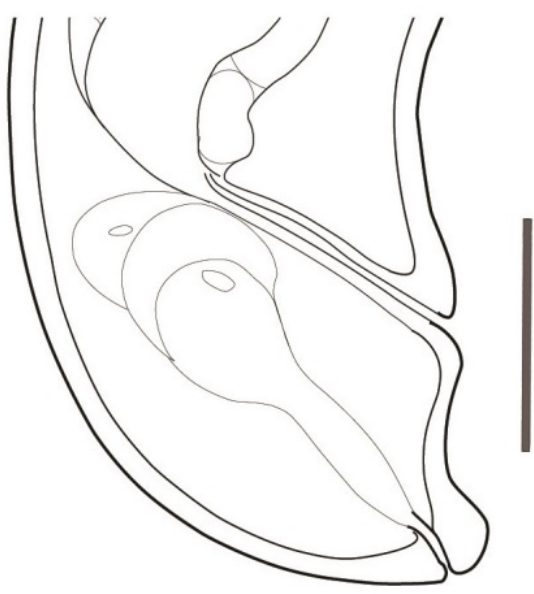

C

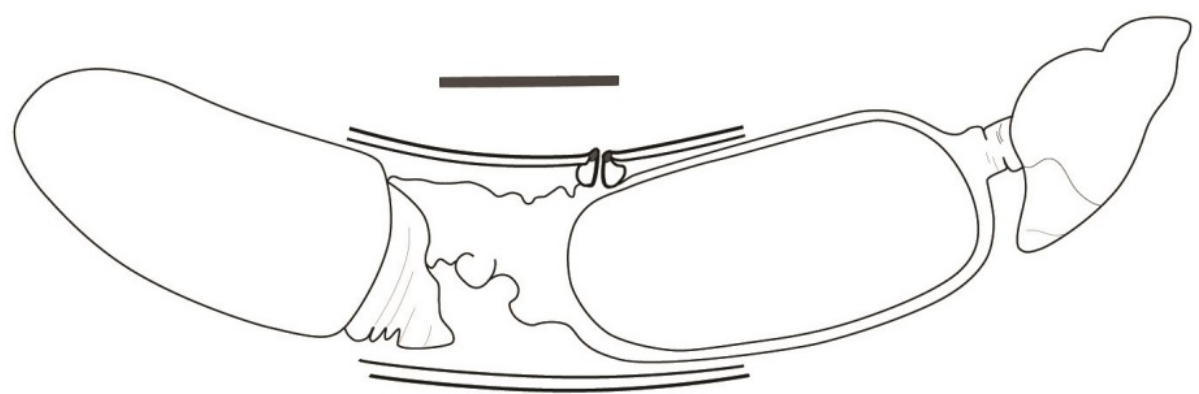

Figure 2. Mylonchulus oceanicus Andrassy, 1986

A. Head region; B. Female tail region; C. Female reproductive system

(Scale bars: A, B $=20 \mu \mathrm{m} ; \mathrm{C}=40 \mu \mathrm{m}$ ) 
Female: Moderately stout nematodes of small size, $1.0-1.2 \mathrm{~mm}$ long. Habitus after fixation ventrally curved, particularly towards posterior end or C-shaped. Cuticle smooth, 1.5$2 \mu \mathrm{m}$ thick. Lip region 24-28 $\mu \mathrm{m}$ wide, slightly offset from the body contour by a depression. Buccal cavity medium size, 25.5-29 $\mu \mathrm{m}$ long and $15-17.5 \mu \mathrm{m}$ wide, funnel shaped, tapering at base; strongly sclerotized. Amphideal fovea cup-shaped. Dorsal tooth very large, claw-like with sharp apex, pointing forward and situated at $80-86 \%$ of buccal cavity length from its base. Small rasp-like denticles arranged in seven transverse rows in ventrosublateral wall. Ventrosublateral teeth absent. Oesophagus cylindrical, 333-369 $\mu \mathrm{m}$ long, surrounding base of buccal cavity. Secretory-excretory pore not observed. Oesophago-intestinal junction nontuberculate. Rectum straight, thick-walled and muscular, 21-24 $\mu \mathrm{m}$, shorter than anal body width. Reproductive system didelphicamphidelphic, both branches equally developed with ovary reflexed. Sphincter between oviduct and uterus absent. Vulva a transverse slit in ventral view, not protruding. Vagina short with pars refringens vaginae sclerotized, visible as 2 pieces in optical section. A large egg in posterior branch, 48x90 $\mu \mathrm{m}$ large. Tail short, plump, ventrally bent, nearly semicircular in its dorsal contour, 19-24 $\mu \mathrm{m}$ or $0.65-0.75$ anal body width. Tail terminus slightly but distinctly dorsal curved, round or obtuse. Three large caudal glands prominent, spinneret present in subdorsal terminus with cuticularized valvular apparatus.

Male: Not found.

Remark: The measurements and description of Vietnamese specimens correspond well with the type population from Hawaii, USA (Andrassy, 1986) [3] and other population from Okinawa, Japan [2] except for slightly shorter tail $(\mathrm{c}=47-56$ vs 39-49).

Locality: Lang Son.

Table 2. Morphometric data of Mylonchulus oceanicus Andrassy, 1986

\begin{tabular}{lccc}
\hline & \multicolumn{3}{c}{ Mylonchulus oceanicus } \\
\cline { 2 - 4 } & $\begin{array}{c}\text { Type population } \\
\text { Hawaii (USA) }\end{array}$ & $\begin{array}{c}\text { Okinawa } \\
\text { (Japan) }\end{array}$ & $\begin{array}{c}\text { Lang Son } \\
\text { (Vietnam) }\end{array}$ \\
\cline { 2 - 4 } & Andrassy (1986) & Ahmad et al. (2010) & Present paper \\
\hline $\mathrm{n}$ & $?+9$ & 6 + & 10 + \\
\hline $\mathrm{L}(\mathrm{mm})$ & $1.06-1.23$ & $0.99-1.44$ & $1.04-1.18$ \\
$\mathrm{a}$ & $24-26$ & $24.6-29.7$ & $20.6-25.4$ \\
$\mathrm{~b}$ & $3-3.2$ & $2.9-3.7$ & $3-3.3$ \\
$\mathrm{c}$ & $34-35$ & $39-49$ & $47.5-56$ \\
$\mathrm{c}$ & $1-1.2$ & $0.7-0.9$ & $0.65-0.75$ \\
$\mathrm{~V}(\%)$ & $63-64$ & $61-66$ & $61.6-66$ \\
Buccal cavity length $(\mu \mathrm{m})$ & $26-29$ & $26-30$ & $25.5-29$ \\
Buccal cavity width $(\mu \mathrm{m})$ & $16-17$ & $17-19$ & $15-17.5$ \\
Apex of dorsal tooth position & $80-82$ & - & $80-86$ \\
from base of buccal cavity $(\%)$ & $25-26$ & $24-30$ & $24.6-28.2$ \\
Lip region width $(\mu \mathrm{m})$ & - & $9-10$ & - \\
Lip region height $(\mu \mathrm{m})$ & $350-410$ & $340-444$ & $333-369$ \\
Neck length $(\mu \mathrm{m})$ & $43-49$ & - & $44-53$ \\
Body diameter at vulva $(\mu \mathrm{m})$ & - & - & $28-34$ \\
Anal body diameter $(\mu \mathrm{m})$ & $31-35$ & $25-34$ & $19-24$ \\
Tail length $(\mu \mathrm{m})$ & - & $21-27$ & $21-24$ \\
Rectum $(\mu \mathrm{m})$ & & & \\
\hline
\end{tabular}

?. no information. 
Currently, 20 species of Mylonchulus genus were recorded and an updated key to species of Mylonchulus genus in Vietnam (based on Ahmad \& Jairajpuri, 2010 [1]) was present as bellow:

1a) Female reproductive system paired, amphidelphic. 2

1b) Female reproductive system unpaired, prodelphic. Post-uterine sac shorter than vulval body diameter or completely absent .....

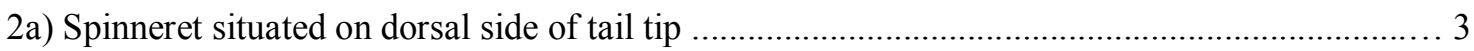

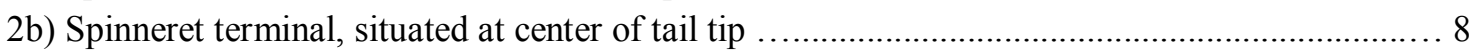

3a) Tail tip dorsally bent; ventro-sublateral teeth absent ................................................. oceanicus

3b) Tail tip not dorsally bent; ventro-sublateral teeth mostly present............................................ 4

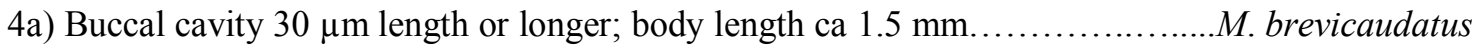

4b) Buccal cavity $20 \mu \mathrm{m}$ length or less than; body length shorter $1.5 \mathrm{~mm}$

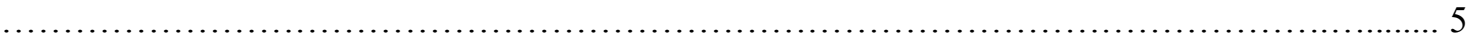

5a) Body length very small, about $0.6-0.7 \mathrm{~mm}$; buccal cavity broad, barrel

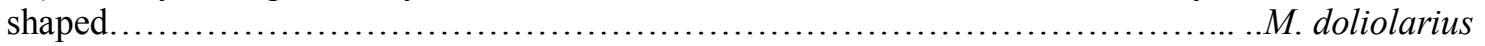

5b) Body longer; buccal cavity as usual, strongly tapering toward its base............................... 6

6a) Ventro-sublateral teeth present, prominent. Tail longer, 35-46 $\mu \mathrm{m}$.......................... brachyuris

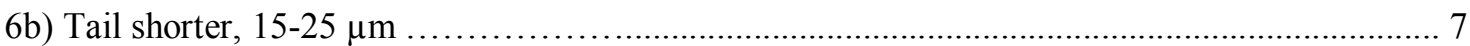

7a) Intestine characteristically narrowed at genital region............................................. contractus

7b) Intestine not strikingly narrowed at genital region, tail tip round .................... nainitalensis

8a) Tail sigmoid, sharply bent ventrad with digitate posterior part inclining slightly dorsally and

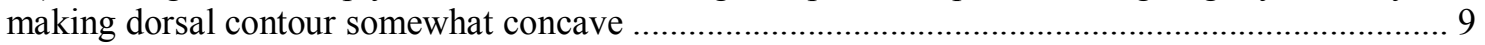

$8 \mathrm{~b})$ Tail not sigmoid, either more or less arcuate or if subdigitate, showing no concave dorsal contour or not sharply bent ventrad ....

11

9a) Ventro-sublateral denticles rasp-like numerous (ca 10-15 irregular rows)............. dentatus

9b) Ventro-sublateral denticles rasp-like, less than 10 rows 10

10a) Ventro-sublateral denticles rasp-like in 6 rows; no advulval papillae ............. kermaniensis

10b) Ventro-sublateral denticles rasp-like in 7-8 rows. Advulval papillae absent M. sigmaturus

11a) Tail longer, 2-4 anal body width 12

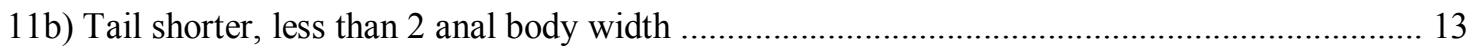

12a) Ventro-sublateral denticles densely arranged, in ca 10 irregular rows .............. apapillatus

12b) Ventro-sublateral denticles not so dense, in 5-7 rows ............................... polonicus

13a) Ventro-sublateral teeth absent ...................................................... amurus

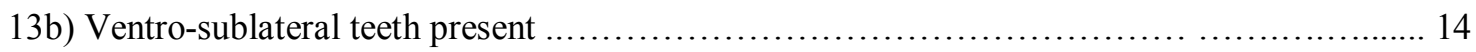

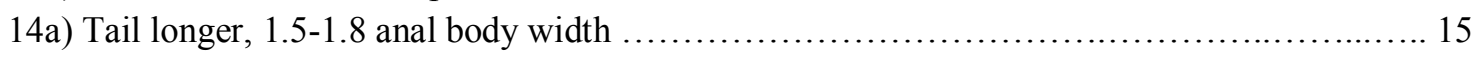

14b) Tail shorter, as long as anal body width .................................................. 16

15a) Tail arcuate, cylindrical for almost entire length. Advulva papillae present .......... M. lacustris

15b) Tail sharply bent ventrad, consisting of an anterior wider and a posterior slender. Rasp-like denticles in 6-7 rows.

M. hawaiiensis

16a) Tail obtuse with bluntly rounded tip ....

M. orbitus

16b) Tail conoid with narrowly rounded tip .....................................................

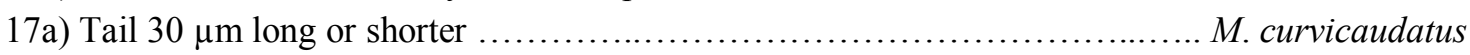

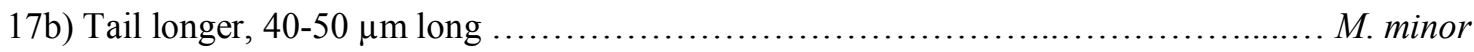


18a) Tail longer, 1.5-2 anal body width. Post uterine sac completely absent M. mulveyi

18b) Tail short, 1 anal body width. Ventro-sublateral denticles in 5-6 transverse rows 19

19a) Caudal spinneret subdorsal M. orientalis

19b) Caudal spinneret terminal M. index

Remarks: Of the total 20 species of the genus Mylonchulus found in Vietnam, eight species are cosmopolitan, viz. M. apapillatus was recorded in India and Korea; M. kermaniensis, from Iran; $M$. amurus and $M$. dentatus, from India, and two species as $M$. doliolarus and M. orientalis from Vietnam.

\section{REFERENCES}

1. Ahmad W., Jairajpuri M. S., 2010. Mononchida: The predaceous nematodes. Brill Leiden-Boston. 298pp.

2. Ahmad W., Mizukubo T., Yoshida M., 2010. Mononchida (nematode) from Japan. Journal of Nematode Morphology and Systematics, 13(2): 123-156.

3. Andrassy I., 1986. Fifteen new nematode species from the southern hemisphere. Acta Zoologica hungarica, 32: 1-33.
4. Nguyen $\mathrm{Vu}$ Thanh, 2007. Dong vat chi Viet Nam. Giun tron song tu do. Monhysterida, Araeolaimida, Chromadorida, Rhabditida, Enoplida, Mononchida, Dorylaimida. Science and Technics Publishing House, Ha Noi. 455pp.

5. Seinhorst J. W., 1959. A rapid method for the transfer of nematodes from fixative to anhydrous glycerin. Nematologica, 4(1): 6769.

6. Shokoohi E., Mehrabi-Nassab A., Mirzaei M., Peneva V., 2013. Study of mononchids from Iran, with description of Mylonchulus kermaniensis sp. n. (Nematode: Mononchida). Zootaxa, 3599(6): 519-534.

7. Southey J. F., 1986. Laboratory methods for work with plant and soil nematodes. London: Her Majesty' Stationery Office. 202pp.

\title{
GHI NHẬN MỚI HAI LOÀI TUYẾN TRÙNG GIỐNG Mylonchulus (Mononchida: Mylonchulidae) VÀ KHÓA ĐỊNH LOẠI CÁC LOÀI CỦA GIỐNG NÀY Ở VIẸTT NAM
}

\author{
Vũ Thị Thanh Tâm
}

Viện Sinh thái và Tài nguyên sinh vật, Viện Hàn lâm KH \& CN Việt Nam

\section{TÓM TẮT}

Hai loài tuyến trùng, Mylonchulus kermaniensis và $M$. oceanicus, được ghi nhận lần đầu tiên và được mô tả cho khu hệ tuyến trùng Việt Nam. M. kermaniensis được ghi nhận từ Nghệ An có số đo và mô tả hoàn toàn phù hợp với số đo và mô tả gốc của loài này từ Iran. Tuy nhiên, không quan sát thấy nhú vulva ở tất cả cá thể từ quần thể ở Việt Nam trong khi đó, có 2 trong số 6 cá thể của quần thể loài này từ Iran có nhú vulva. $M$. oceanicus được ghi nhận từ Lạng Sơn có số đo và mô tả phù hợp với số đo và mô tả gốc của loài này từ Hawaii, Hoa Kỳ cũng như quần thể từ Nhật Bản ngoại trừ đuôi hơi ngắn hơn $(\mathrm{c}=47-56$ so với $\mathrm{c}=34-35$ ở quần thể gốc và $\mathrm{c}=39-49$ ở quần thể Nhật Bản)

Như vậy, cho đến nay đã có 20 loài thuộc giống Mylonchulus được ghi nhận ở Việt Nam và lần đầu tiên đưa ra khóa định loại cho tất cả các loài của giống này được ghi nhận ở Việt Nam.

Tù khóa: Mylonchulus, ghi nhận mới, tuyến trùng, Việt Nam.

Received 23 March 2016, accepted 20 September 2016 